joints to which the human reader and our system assigned the same score) for MCP joints was $80.5 \%$, that for PIP joints was $72.3 \%$. In only $1.8 \%$ (MCPs) and $1.7 \%$ (PIPs) of cases did the predicted score differ by more than one point from the ground truth (Fig. 2).
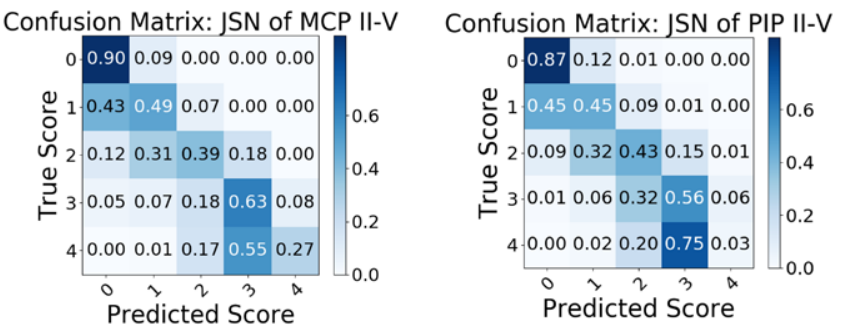

Fig. 2. Confusion matrices of automatically assigned scores ('predicted score') vs. the human reader ground truth ('true score').

Conclusion: Although a number of previous efforts have been published, none have succeeded in replacing manual scoring systems at scale. To our knowledge, this is the first work that utilizes a dataset of adequate size to apply deep learning to automate JSN scoring. Our results are, even in this early version, in good agreement with human reader ground truth scores. In future versions, this system can be expanded to the detection of erosions and to all joints contained in the SvdH score.

References:

[1] Boini, S. \& Guillemin, F. Radiographic scoring methods as outcome measures in rheumatoid arthritis: properties and advantages. Ann. Rheum. Dis. 60, 817-827 (2001).

[2] van der Heijde, D. How to read radiographs according to the Sharp/van der Heijde method. J. Rheumatol. 27, 261-263 (2000).

[3] Payer, C., Štern, D., Bischof, H. \& Urschler, M. Regressing Heatmaps for Multiple Landmark Localization Using CNNs. in Medical Image Computing and Computer-Assisted Intervention - MICCAI 2016 230-238 (Springer, Cham, 2016). doi:10.1007/978-3-319-46723-8_27.

[4] Simonyan, K. \& Zisserman, A. Very Deep Convolutional Networks for LargeScale Image Recognition. arXiv:1409.1556 [cs] (2015).

[5] Huang, G., Liu, Z., van der Maaten, L. \& Weinberger, K. Q. Densely Connected Convolutional Networks. arXiv:1608.06993 [CS] (2016).

Disclosure of Interests: Thomas Deimel: None declared, Daniel Aletaha Grant/research support from: AbbVie, Novartis, Roche, Consultant of: AbbVie, Amgen, Celgene, Lilly, Medac, Merck, Novartis, Pfizer, Roche, Sandoz, Sanofi Genzyme, Speakers bureau: AbbVie, Celgene, Lilly, Merck, Novartis, Pfizer, Sanofi Genzyme, UCB, Georg Langs Shareholder of: Co-Founder/Shareholder contextflow $\mathrm{GmbH}$, Grant/research support from: Grants by Novartis, Siemens Healthineers, NVIDIA

DOI: 10.1136/annrheumdis-2020-eular.714

\section{OP0060 MACHINE LEARNING BASED BERLIN SCORING OF MAGNETIC RESONANCE IMAGES OF THE SPINE IN PATIENTS WITH ANKYLOSING SPONDYLITIS FROM THE MEASURE 1 STUDY}

A. Jamaludin ${ }^{1}$, R. Windsor ${ }^{1}$, S. Ather ${ }^{1}$, T. Kadir ${ }^{1}$, A. Zisserman ${ }^{1}$, J. Braun ${ }^{2}$, L. S. Gensler ${ }^{3}$, P. Machado ${ }^{4}$, M. Ǿstergaard ${ }^{5}$, D. Poddubnyy ${ }^{6}$, T. Coroller $^{7}$, B. Porter ${ }^{8}$, S. Mpofu ${ }^{9}$, A. Readie ${ }^{8}{ }^{1}$ University of Oxford, Oxford, United Kingdom; ${ }^{2}$ Ruhr University Bochum, Herne, Germany; ${ }^{3}$ University of California, San Francisco, United States of America; ${ }^{4}$ University College London, London, United Kingdom; ${ }^{5}$ University of Copenhagen, Copenhagen, Denmark; ${ }^{6}$ Charité Universitätsmedizin Berlin, Berlin, Germany; ${ }^{7}$ Novartis Pharmaceuticals Corporation, East Hanover, United States of America; ${ }^{8}$ Novartis Pharmaceuticals Corporation, East Hanover, United States of America; ${ }^{9}$ Novartis Pharma AG, Basel, Switzerland

Background: Magnetic resonance imaging (MRI) offers a non-invasive and objective method of early diagnosis and classification, monitoring disease burden and treatment response for patients (pts) with axial spondyloarthritis (axSpA) including ankylosing spondylitis (AS). ${ }^{1}$ Numerous scoring schemes such as the AS Spine MRI Activity (ASspiMRla) score are available for the quantitative assessment of MRI, but are subject to intra- and inter-rater variability, labor intensive and costly. Nevertheless, quantification of MRI changes has become an important tool to demonstrate treatment success of biologic drugs in axSpA.

Objectives: To evaluate the performance of machine learning (ML) based software for automated Berlin grading of spinal MRI bone marrow oedema in pts with AS and compare with expert scoring
Methods: Fully automated ML software (Figure) was developed to detect and label 23 vertebrae, define vertebral units (VU) as per the Berlin modification of the ASspiMRla score, and score each VU as either 0 (score of 0 ) or 1 (score of 1,2 or 3 ). The ML algorithm was based on the previously developed SpineNet software. ${ }^{2}$ Analysis included 108 pts from the secukinumab MEASURE 1 study $^{3}$ in which imaging was done using T1 and STIR sagittal MRI at baseline and Weeks 16, 52, 104, 156 and 208. Two expert readers, blinded to treatment and visit, evaluated all images by ASspiMRla score. The scores from Reader 2 (R2) were binned into two groups: 0 vs 1,2 , or 3 . As a result of multiple pt time points and expert reading sessions, the complete dataset comprised of 10,988 VU. Tenway cross-validation at per-VU was used to train and validate the ML software. The dataset was split into 10 randomly selected subsets, ensuring that each pt appears in only one subset, after which 8 subsets were used for training the $\mathrm{ML}$ software, 1 was used to check for correct training and 1 was used for validation. The process was repeated ten times such that all 10 subsets were used for validation. Accuracy weighted for the frequency of each category, sensitivity and specificity were calculated using scores from R2 as reference. Intra-reader accuracy was also calculated

Results: Accuracy of the software in relation to expert reader scores was $67 \%$ with a sensitivity of 0.63 and specificity of 0.70 . The intra-reader accuracy was $71 \%$ and $77 \%$ for R1 and R2, respectively. Individual VU scoring of the Software vs. R2 are presented in the Table as a confusion matrix.

Conclusion: Automated scoring of MR images in AS pts provided moderate agreement to that of expert reader-based assessments. ML software has potential to provide an automated guided-reading approach to scoring MR images, which may enable further clinical insights.

References:

[1] Lukas C, et al. J Rheumatol. 2007;34:862-70.

[2] Jamaludin A, et al. Eur Spine J. 2017;26:1374-83.

[3] Baeten D, et al. N Engl J Med. 2015;373,2534-48

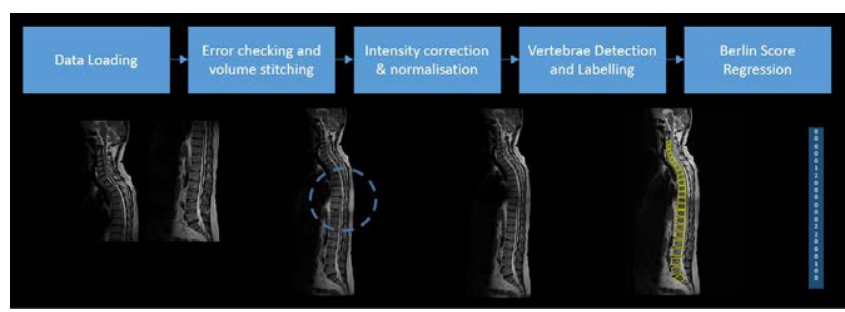

Figure. Processing pipeline of automated Berlin scoring software

Table. Confusion matrix between the software and R2

\begin{tabular}{lccc}
\hline & $\begin{array}{c}\text { Software } \\
\text { Score }=\mathbf{0}\end{array}$ & $\begin{array}{c}\text { Software } \\
\text { Score }=\mathbf{1 , 2} \text { or 3 }\end{array}$ & Total VU scored \\
\hline R2 Score $=0$ & $7199(70 \%)$ & $3068(30 \%)$ & 10,267 \\
R2 Score $=1,2$ or 3 & $251(35 \%)$ & $475(65 \%)$ & 726 \\
& 7,450 & 3,543 & 10,993 \\
\hline
\end{tabular}

Percentages calculated as a fraction over the total in each row. Overall accuracy is the average of the highlighted percentages.

Disclosure of Interests: Amir Jamaludin: None declared, Rhydian Windsor: None declared, Sarim Ather: None declared, Timor Kadir: None declared, Andrew Zisserman: None declared, Juergen Braun Grant/research support from: Abbvie (Abbott), Amgen, BMS, Boehringer, Celgene, Celltrion, Centocor, Chugai, Eli Lilly and Company, Medac, MSD (Schering Plough), Mundipharma, Novartis Pfizer (Wyeth), Roche, Sanofi- Aventis, and UCB Pharma, Consultant of: Abbvie (Abbott), Amgen, BMS, Boehringer, Celgene, Celltrion, Centocor, Chugai, EBEWE Pharma, Eli Lilly and Company, Medac, MSD (Schering-Plough), Mundipharma, Novartis, Pfizer (Wyeth), Roche, Sanofi-Aventis, and UCB Pharma, Speakers bureau: Abbvie (Abbott), Amgen, BMS, Boehringer, Celgene, Celltrion, Centocor, Chugai, EBEWE Pharma, Eli Lilly and Company, Medac, MSD (Schering-Plough), Mundipharma, Novartis, Pfizer (Wyeth), Roche, Sanofi-Aventis, and UCB Pharma, Lianne S. Gensler Grant/research support from: Pfizer, Novartis, UCB, Consultant of: AbbVie, Eli Lilly, GSK, Novartis, UCB, Pedro Machado Consultant of: Abbvie, Celgene, Janssen, Lilly, MSD, BMS, Novartis, Pfizer, Roche and UCB, Speakers bureau: AbbVie, Centocor, Eli Lilly, Janssen, MSD, Novartis Pfizer and UCB Pharma, Mikkel Ǿstergaard Grant/research support from: AbbVie, Bristol-Myers Squibb, Celgene, Merck, and Novartis, Consultant of: AbbVie Bristol-Myers Squibb, Boehringer Ingelheim, Celgene, Eli Lilly, Hospira, Janssen, Merck, Novartis, Novo Nordisk, Orion, Pfizer, Regeneron, Roche, Sandoz, Sanofi, and UCB, Speakers bureau: AbbVie, Bristol-Myers Squibb, Boehringer Ingelheim, Celgene, Eli Lilly, Hospira, Janssen, Merck, Novartis, Novo Nordisk, Orion, Pfizer, Regeneron, Roche, Sandoz, Sanofi, and UCB, Denis Poddubnyy 
Grant/research support from: AbbVie, MSD, Novartis, and Pfizer, Consultant of: AbbVie, Bristol-Myers Squibb, Eli Lilly, MSD, Novartis, Pfizer, Roche, UCB, Speakers bureau: AbbVie, Bristol-Myers Squibb, Eli Lilly, MSD, Novartis, Pfizer, Roche, UCB, Thibaud Coroller Shareholder of: Novartis, Employee of: Novartis, Brian Porter Shareholder of: Novartis, Employee of: Novartis, Shephard Mpofu Shareholder of: Novartis, Employee of: Novartis, Aimee Readie Shareholder of: Novartis, Employee of: Novartis DOI: 10.1136/annrheumdis-2020-eular.1207

\section{OP0061 FEASIBILITY STUDY ON AN AUTOMATED QUANTITATIVE SYSTEM FOR ULTRASOUND JOINT INFLAMMATION ASSESSMENT IN RHEUMATOID ARTHRITIS USING DEEP LEARNING}

Y. K. $\operatorname{Tan}^{1}$, S. Suriyanto ${ }^{2}$, P. H. Yeung ${ }^{3}$, S. Xu $\mathbf{u}^{4,5} .{ }^{1}$ Department of Rheumatology and Immunology, Singapore General Hospital, Singapore, Singapore; ${ }^{2}$ Diagnostics Development Hub, Accelerate Technologies, Singapore, Singapore; ${ }^{3}$ Department of Engineering Science, Institute of Biomedical Engineering, University of Oxford, Oxford, United Kingdom; ${ }^{4}$ Department of General Surgery, Nanfang Hospital, Southern Medical University, Guangzhou, China; ${ }^{5}$ Voxel Imaging Pte Ltd, Singapore, Singapore

Background: The most widely accepted ultrasound (US) joint inflammation scoring system in rheumatoid arthritis (RA) is semi-quantitative in nature. This process involves manual image acquisition followed by image interpretation. The subjectivity inherent in manual scoring may be overcome by the development of an automated quantitative system to measure joint inflammation.

Objectives: To develop an automated quantitative system to measure US detected power Doppler (PD) joint inflammation in patients with RA.

Methods: The synovial region of interest (sROI) on US images at the metacarpophalangeal joints (MCPJs) and the metatarsophalangeal joint (MTPJs) within the Doppler box is manually segmented by a clinician experienced in musculoskeletal US (figure 1). PD joint inflammation was scored manually semi-quantitatively (0-3). Deep learning based image segmentation was applied to the US images to automatically identify sROI and quantify the amount of PD signals within the SROI (figure 1) to obtain a computer derived PD reading reflecting the extent of PD vascularity within the sROI. The performance of computer derived PD reading was evaluated in comparison with the clinician's manual scoring.

Results: 820 joints from bilateral $1^{\text {st }}$ to $5^{\text {th }}$ MCPJs and MTPJs in 41 adult RA patients (baseline characteristics: $75.6 \%$ Chinese; $73.2 \%$ female; mean (SD) DAS28, 4.23 (1.25); mean (SD) disease duration, 73.3 (57.8) months) were evaluated in this cross-sectional study. The respective mean (SD)/ median (IQR) computer derived PD readings were $0.13(0.75) / 0.04(0.08), 1.62(1.77) / 1.21$ (1.19) and $10.12(6.86) / 7.51$ (5.24) for manual score 0,1 and 2 (no joints had manual score 3), with statistically significant differences found among the different manual score classes (for non-normally distributed data, Kruskal-Wallis

Figure 1. Manual and automated segmentation of synovial region of interest

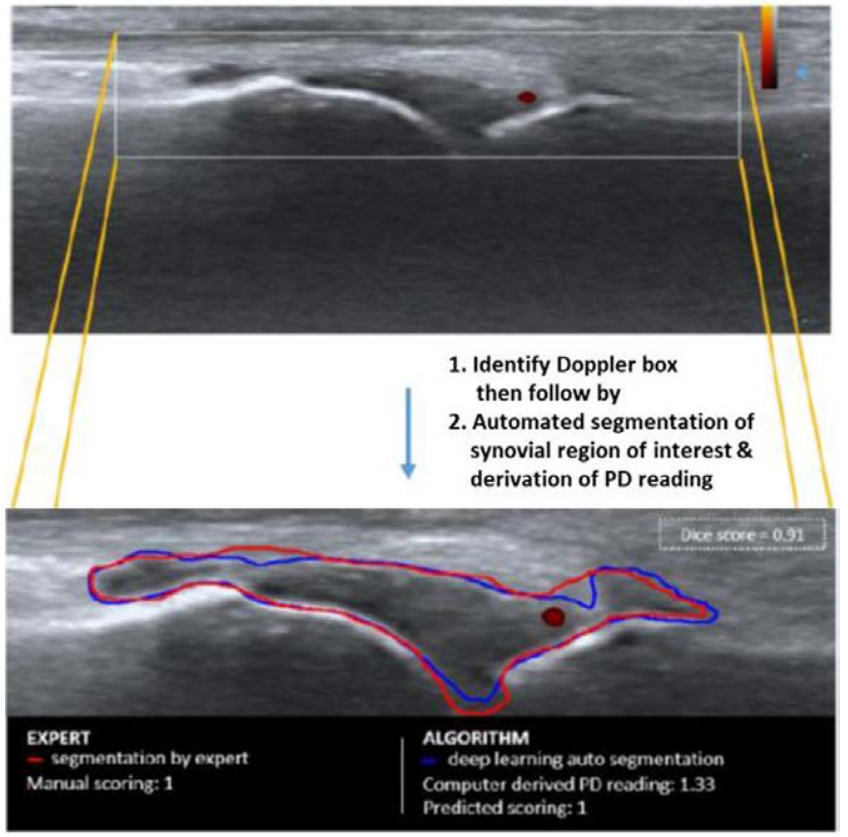

Table 1. Performance of computer prediction versus clinician evaluation

\section{Score 0 vs. 1}

Assessor

Computer Prediction: Score 0

Computer Prediction: Score 1

Sensitivity $=99.14 \%$,

Specificity $=97.00 \%$

Score 1 vs. 2

Assessor

Computer Prediction: Score 1

Computer Prediction: Score 2

Sensitivity $=97.14 \%$

Specificity $=93.97 \%$
Clinician Evaluation: Score 0 Clinician Evaluation: Score 1 615
19
115

Clinician Evaluation: Score 1 Clinician Evaluation: Score 2

$\begin{array}{cc}109 & 2 \\ 7 & 68\end{array}$
H-test, $p=1.69 \times 10^{-92}$, Mann-Whitney Test: manual score 0 versus $1, p=1.04$ x $10^{-62}$; manual score 0 versus $2, p=3.28 \times 10^{-43}$; manual score 1 versus 2 , $\mathrm{p}=1.53 \times 10^{-28}$ ). Area under the ROC curve (AUC) based on computer derived PD reading cut-off of 0.26 to identify manual score 0 versus 1 was 0.98 , while AUC based on computer derived PD reading cut-off of 3.37 to identify manual score 1 versus 2 was 0.98 . The overall agreement of the score classes $(0,1$ and 2) based on computer prediction using the above cut-offs versus manual scores of 0,1 and 2 is $791 / 820=96.46 \%$. Table 1 summarizes the performance of computer prediction using the above cut-offs when compared to clinician evaluation (i.e. score 0 versus 1, comparing computer prediction with clinician evaluation, sensitivity $=99.14 \%$ and specificity $=97.00 \%$; score 1 versus 2 , comparing computer prediction with clinician evaluation, sensitivity $=97.14 \%$ and specificity=93.97\%).

Conclusion: An automated quantitative system for US PD joint inflammation assessment using deep learning showed high sensitivity and specificity when results from computer prediction were compared to clinician evaluation. Further validation in a larger RA cohort with a longitudinal study design would be required.

References: Nil

Disclosure of Interests: None declared

DOI: 10.1136/annrheumdis-2020-eular.1501

\section{OP0062 PREDICTIVE VALUE OF BONE TEXTURE FEATURES EXTRACTED BY DEEP LEARNING MODELS FOR THE DETECTION OF OSTEOARTHRITIS: DATA FROM THE OSTEOARTHRITIS INITIATIVE}

C. F. Kuo ${ }^{1,2}$, K. Zheng ${ }^{3}$, S. Miao ${ }^{3}$, L. Lu ${ }^{3}$, C. I. Hsieh ${ }^{1}$, C. Lin ${ }^{2}$, T. Y. Fan ${ }^{2} .{ }^{1}$ Chang Gung Memorial Hospital, Division of Rheumatology, Allergy and Immunology, Taoyuan, Taiwan, Republic of China; ${ }^{2}$ Chang Gung Memorial Hospital, Center for Artificial Intelligence in Medicine, Taoyuan, Taiwan, Republic of China; ${ }^{3}$ PAII labs, Bethesda, United States of America

Background: Osteoarthritis is a degenerative disorder characterized by radiographic features of asymmetric loss of joint space, subchondral sclerosis, and osteophyte formation. Conventional plain films are essential to detect structural changes in osteoarthritis. Recent evidence suggests that fractal- and entropy-based bone texture parameters may improve the prediction of radiographic osteoarthritis. ${ }^{1}$ In contrast to the fixed texture features, deep learning models allow the comprehensive texture feature extraction and recognition relevant to osteoarthritis.

Objectives: To assess the predictive value of deep learning-extracted bone texture features in the detection of radiographic osteoarthritis.

Methods: We used data from the Osteoarthritis Initiative, which is a longitu dinal study with 4,796 patients followed up and assessed for osteoarthritis. We used a training set of 25,978 images from 3,086 patients to develop the textual model. We use the BoneFinder software ${ }^{2}$ to do the segmentation of distal femur and proximal tibia. We used the Deep Texture Encoding Network (Deep-TEN) ${ }^{3}$ to encode the bone texture features into a vector, which is fed to a 5-way linear classifier for Kellgren and Lawrence grading for osteoarthritis classification. We also developed a Residual Network with 18 layers (ResNet18) for comparison since it deals with contours as well. Spearman's correlation coefficient was used to assess the correlation between predicted and reference $\mathrm{KL}$ grades. We also test the performance of the model to identify osteoarthritis (KL grade $\geq 2$ ).

Results: We obtained 6,490 knee radiographs from 446 female and 326 male patients who were not in the training sets to validate the performance of the models. The distribution of the $\mathrm{KL}$ grades in the training and testing sets were shown in Table 1. The Spearman's correlation coefficient was 0.60 for the Deep-TEN and 0.67 for the ResNet18 model. Table 2 shows the performance of the models to detect osteoarthritis. The positive predictive value for Deep-TEN and ResNet18 model classification for OA was $81.37 \%$ and $87.46 \%$, respectively. 